\title{
Pairs of additive quadratic forms modulo one
}

\author{
by \\ R. C. BAKer (Egham) and S. Schäffer (Löhne)
}

1. Introduction. Let $\|x\|$ denote the distance from $x$ to the nearest integer. Let $\varepsilon>0$. A well-known theorem of Heilbronn [15] states that for $N>C_{1}(\varepsilon)$, and any real number $\alpha$, we have

$$
\min _{1 \leq n \leq N}\left\|\alpha n^{2}\right\|<N^{-1 / 2+\varepsilon} .
$$

Among many possible extensions, the following was considered by Danicic [9]. We seek a positive number $\alpha(s)$ with the following property:

Let $Q\left(x_{1}, \ldots, x_{s}\right)$ be a real quadratic form, then for $N>C_{2}(s, \varepsilon)$ we have

$$
\left\|Q\left(n_{1}, \ldots, n_{s}\right)\right\|<N^{-\alpha(s)+\varepsilon}
$$

for some integers $n_{1}, \ldots, n_{s}$,

$$
0<\max \left(\left|n_{1}\right|, \ldots,\left|n_{s}\right|\right) \leq N .
$$

Danicic was able to take $\alpha(s)=s /(s+1)$. An important step forward occurred when Schinzel, Schlickewei and Schmidt [18] showed the relevance of the following "discrete version" of the problem. We seek the least positive number $B_{s}(q)$ with the following property.

For any $K_{i}>0$ satisfying

$$
\left(K_{1} \ldots K_{s}\right)^{1 / s} \geq B_{s}(q),
$$

and any quadratic form $Q$ with integer coefficients, the congruence $Q(\boldsymbol{x}) \equiv 0$ $(\bmod q)$ has a nonzero solution satisfying

$$
\left|x_{i}\right| \leq K_{i} \quad(1 \leq i \leq n) .
$$

Further work on this problem was done by Baker and Harman [6] and by Heath-Brown [14]. Heath-Brown showed that

$$
B_{s}(q)<C_{3}(s, \varepsilon) q^{\beta(s)+\varepsilon}
$$

where $\beta(4)=5 / 8, \beta(6)=15 / 26, \beta(8)=6 / 11, \beta(10)=\beta(11)=8 / 15$ and $\beta(s)=1 / 2+3 / s^{2}$ for even $s \geq 12$. For $s=3,5,7$ the exponent 
$\beta(s)=1 / 2+1 /(2 s)[6]$ remains the best known. By arguing as in [6], one can show that the exponent

$$
\alpha(s)=\frac{s}{2+s \beta(s)}
$$

is permissible in (1.1), whenever (1.3) holds.

Not surprisingly, one can do better for real additive quadratic forms. It is convenient for applications to seek solutions in a box rather than a cube.

Theorem 1. Suppose that (1.3) holds. Let $\sigma(1)=1 / 2, \sigma(2)=1$,

$$
\sigma(s)=\frac{s}{2+(s-2) \beta(s)} \quad(s \geq 3) .
$$

Let $Q\left(x_{1}, \ldots, x_{s}\right)$ be an additive quadratic form. Let $N>C_{4}(s, \varepsilon)$. Given positive $N_{1}, \ldots, N_{s}$ with

$$
N_{1} \ldots N_{s} \geq N^{s}
$$

there exist non-negative integers $n_{1}, \ldots, n_{s}$ not all zero satisfying $n_{i} \leq N_{i}$ $(i=1, \ldots, s)$ and

$$
\left\|Q\left(n_{1}, \ldots, n_{s}\right)\right\|<N^{-\sigma(s)+\varepsilon} .
$$

The case $s=2$ of Theorem 1 is a generalization of a theorem of Cook [7]. For $s \geq 3$, see [13] and [1] for earlier results along the lines of Theorem 1 .

In proving Theorem 1 we assume, as we may, that $1 / 2 \leq \beta(s) \leq 1 / 2+$ $1 /(2 s-4)$.

We apply Theorem 1 to pairs of additive forms.

Theorem 2. Define $\sigma(s)$ as above. Let $Q_{1}\left(x_{1}, \ldots, x_{s}\right), Q_{2}\left(x_{1}, \ldots, x_{s}\right)$ be additive quadratic forms. Then for $N>C_{5}(s, \varepsilon)$ we have

$$
\max \left(\left\|Q_{1}(\boldsymbol{n})\right\|,\left\|Q_{2}(\boldsymbol{n})\right\|\right)<N^{-\tau(s)+\varepsilon}
$$

for some integers $n_{1}, \ldots, n_{s}$ satisfying (1.2). Here

$$
\begin{gathered}
\tau(2)=1 / 3, \quad \tau(3)=3 / 7, \quad \tau(4)=1 / 2 ; \\
\tau(s)= \begin{cases}s \sigma(s) /(8 \sigma(s)+2 s-8) & \text { for } 5 \leq s \leq 7, \\
\sigma(s) /(1+\sigma(s)) & \text { for } s \geq 8 .\end{cases}
\end{gathered}
$$

Since $\sigma(s)$ has limit 2 as $s \rightarrow \infty$, we see that $\tau(s)$ has limit $2 / 3$. However, we can replace $\tau(s)$ by an exponent whose limit is 1 ; see Baker and Harman [5]. In fact, the method of [5] may be refined to give an improvement of Theorem 2 for $s \geq 24$.

For earlier results in a small number of variables along the lines of Theorem 2, see Liu [17] and Baker and Gajraj [4]. The exponent in [4] is much poorer, namely $-1 / 5+\varepsilon$ for $s \geq 2$. This is partly because we now have at our disposal the "lattice method" of Schmidt [19], whose result may be 
stated as

$$
\tau(1)=1 / 6 \text {. }
$$

Weaker versions of this last result were found earlier by Danicic [8], [10] and Liu [16].

For arbitrary pairs of quadratic forms, the first results analogous to (1.5) were given by Danicic [11]. Recently Baker and Brüdern [3] improved these results. For example, the analogue of (1.5) for a pair of binary forms has $1 / 5$ in place of $\tau(2)$. Once again, [5] is stronger for large $s$.

Throughout the paper, implied constants depend at most on $\varepsilon, s$. We write $e(\theta)=e^{2 \pi i \theta}$. The cardinality of a finite set $\mathcal{A}$ is denoted by $|\mathcal{A}|$.

2. Proof of Theorem 1. We require two lemmas from [2].

Lemma 1. Let $x_{j}(j=1, \ldots, N)$ be real numbers satisfying $\left\|x_{j}\right\| \geq M^{-1}$. Then

$$
\sum_{m \leq M}\left|\sum_{n=1}^{N} e\left(m x_{n}\right)\right|>N / 6 .
$$

Proof. This is Theorem 2.2 of [2].

Lemma 2. Let $\delta>0$ and $N>C_{6}(\delta)$. Let $\alpha$ be real. Let $L$ be a natural number such that

$$
L^{\delta}<N
$$

If

$$
\sum_{m=1}^{L}\left|\sum_{n=1}^{N} e\left(m \alpha n^{2}\right)\right|^{2}>A
$$

where $A \geq N^{1+\delta} L$, then there exist coprime integers $r$ and $s$ with $r \leq$ $L N^{2+\delta} A^{-1}$ and

$$
|\alpha r-s|<N^{\delta} A^{-1}
$$

Proof. This is given in all essentials in [2], although the condition (2.2) is weaker than the corresponding inequality in [2].

Our next step is to prove Theorem 1 under the additional restrictions

$$
s \geq 2 ; \quad N_{j} \geq N^{\varepsilon / 4} \quad(j=1, \ldots, s) .
$$

LEMma 3. The assertion of Theorem 1 is true when (2.5) holds.

Proof. Suppose that the assertion is false. Then, by Lemma 1,

$$
\sum_{m=1}^{M} T_{1}(m) \ldots T_{s}(m) \geq C_{7}(s) N_{1} \ldots N_{s} .
$$


Here $M=1+\left[N^{\sigma(s)-\varepsilon}\right], Q(\boldsymbol{x})=\alpha_{1} x_{1}^{2}+\ldots+\alpha_{s} x_{s}^{2}$,

$$
T_{j}(m)=\left|\sum_{n \leq N_{j}} e\left(m \alpha_{j} n^{2}\right)\right| .
$$

The contribution from those $m$ in $(2.6)$ having $T_{j}(m)<N^{-2}$ for some index $j$ is negligible. We cover $\left[N^{-2}, C_{7} N_{1} \ldots N_{s}\right]$ by $O(\log N)$ subintervals of the type $[A, 2 A)$. There must exist numbers $A_{j} \geq N^{-2}(j=1, \ldots, s)$ and a subset $\mathcal{B}$ of $[1, M) \cap \mathbb{Z}$ having

$$
\begin{gathered}
A_{j} \leq T_{j}(m)<2 A_{j} \quad(j=1, \ldots, s), \\
\sum_{m \in \mathcal{B}} T_{1}(m) \ldots T_{s}(m) \gg N_{1} \ldots N_{s} /(\log N)^{s} .
\end{gathered}
$$

This implies

$$
|\mathcal{B}|^{2} A_{1}^{2} \ldots A_{s}^{2} \gg N_{1}^{2} \ldots N_{s}^{2}(\log N)^{-2 s} .
$$

We may suppose $\varepsilon$ is sufficiently small. Writing $\delta=\varepsilon^{2}$,

$$
|\mathcal{B}|^{s} A_{1}^{2} \ldots A_{s}^{2} \geq|\mathcal{B}|^{s-2} N_{1}^{2} \ldots N_{s}^{2} N^{-\delta} .
$$

Choose $j, 1 \leq j \leq s$. The inequality

$$
|\mathcal{B}| A_{j}^{2} \leq M N_{j}^{1+\delta}
$$

must be satisfied. Otherwise,

$$
\sum_{m=1}^{M} T_{j}(m)^{2} \geq M N_{j}^{1+\delta} .
$$

Now $M^{\delta} \leq N^{\varepsilon / 4} \leq N_{j}$. Since $N_{j}$ is large, Lemma 2 yields a natural number $r$ such that

$$
\begin{gathered}
r \leq M N_{j}^{2+\delta}\left(M N_{j}^{1+\delta}\right)^{-1}=N_{j}, \\
\left\|\alpha_{j} r^{2}\right\| \leq r\left\|\alpha_{j} r\right\|<N_{j}^{1+\delta}\left(M N_{j}^{1+\delta}\right)^{-1}=M^{-1},
\end{gathered}
$$

contradicting our hypothesis. This proves (2.10).

From (2.10),

$$
|\mathcal{B}|^{s} A_{1}^{2} \ldots A_{s}^{2} \leq M^{s}\left(N_{1} \ldots N_{s}\right)^{1+\delta} .
$$

Suppose first that $s=2$. Then

$$
|\mathcal{B}|^{2} A_{1}^{2} A_{2}^{2} \leq M^{2}\left(N_{1} N_{2}\right)^{1+\delta} .
$$

Combining this with (2.8), (1.4), we have

$$
\begin{gathered}
N_{1}^{2} N_{2}^{2}(\log N)^{-4} \ll M^{2}\left(N_{1} N_{2}\right)^{1+\delta}, \\
M^{2} \gg N^{2-2 \delta}(\log N)^{-4} .
\end{gathered}
$$

This contradicts the definition of $M$, and Lemma 3 is proved for $s=2$. 
Suppose now $s>2$. We combine (2.9) and (2.11) to obtain an upper bound for $|\mathcal{B}|$ :

$$
\begin{gathered}
|\mathcal{B}|^{s-2}\left(N_{1} \ldots N_{s}\right)^{2} N^{-\delta} \leq M^{s}\left(N_{1} \ldots N_{s}\right)^{1+\delta} \\
|\mathcal{B}|^{s-2} \leq M^{s}\left(N_{1} \ldots N_{s}\right)^{-1+\delta} N^{\delta} \leq\left(M N^{-1}\right)^{s} N^{(s+1) \delta}
\end{gathered}
$$

from (1.4).

Choose any $m \in \mathcal{B}$. For any $j \leq s$ for which

$$
A_{j} \geq N_{j}^{1 / 2+\delta},
$$

we apply the case $L=1$ of Lemma 2 . This yields integers $r_{j}, b_{j}$ satisfying

$$
\begin{aligned}
1 & \leq r_{j} \leq\left(N_{j} / A_{j}\right)^{2} N_{j}^{\delta}, \\
\left|m \alpha_{j} r_{j}^{2}-b_{j}\right| & \leq r_{j}\left\|m \alpha_{j} r_{j}\right\| \leq\left(N_{j} / A_{j}\right)^{4} N_{j}^{4 \delta-2} .
\end{aligned}
$$

If (2.13) fails, the last expression in (2.15) is at least 1 , and we can trivially satisfy (2.14) and

$$
\left|m \alpha_{j} r_{j}^{2}-b_{j}\right| \leq\left(N_{j} / A_{j}\right)^{4} N^{4 \delta-2} .
$$

By (2.9), (2.12) and (1.4),

$$
\begin{aligned}
A_{1}^{2} \ldots A_{s}^{2}\left(N_{1} \ldots\right. & \left.N_{s}\right)^{-1-3 \delta}(m / M)^{s / 2} \\
& \geq|\mathcal{B}|^{-2} N^{-\delta}\left(N_{1} \ldots N_{s}\right)^{1-3 \delta}(m / M)^{s / 2} \\
& \geq N^{s-6 s \delta}\left(M N^{-1}\right)^{-2 s /(s-2)}(m / M)^{s / 2} .
\end{aligned}
$$

By the definition of $M$, the last expression in (2.17) is at least $m^{s \beta(s)+2 s \delta}$. Thus

$$
K_{1} \ldots K_{s} \geq C_{3}(s, \delta)^{s} m^{s \beta(s)+s \delta},
$$

where $K_{j}=A_{j}^{2} N_{j}^{-1-3 \delta}(m / M)^{1 / 2}$.

We apply (1.3). There are integers $x_{1}, \ldots, x_{s}$, not all zero, satisfying

$$
\begin{gathered}
\sum_{j=1}^{s} b_{j} x_{j}^{2} \equiv 0(\bmod m), \\
0 \leq x_{j} \leq K_{j} \quad(j=1, \ldots, s) .
\end{gathered}
$$

Taking $n_{j}=r_{j} x_{j}$ we have, by (2.14) and (2.19),

$$
0 \leq n_{j} \leq\left(N_{j} / A_{j}\right)^{2} N_{j}^{\delta} A_{j}^{2} N_{j}^{-1-3 \delta}(m / M)^{1 / 2} \leq N_{j} .
$$

Not all $n_{j}$ are 0 . Moreover,

$$
\sum_{j=1}^{s} \alpha_{j} n_{j}^{2}=\sum_{j=1}^{s} x_{j}^{2} \alpha_{j} r_{j}^{2}=m^{-1} \sum_{j=1}^{s} b_{j} x_{j}^{2}+m^{-1} \sum_{j=1}^{s} x_{j}^{2}\left(\alpha_{j} m r_{j}^{2}-b_{j}\right) .
$$


By (2.18), (2.19) and (2.16),

$$
\begin{aligned}
\left\|\sum_{j=1}^{s} \alpha_{j} n_{j}^{2}\right\| & \leq m^{-1} \sum_{j=1}^{s} x_{j}^{2}\left|\alpha_{j} m r_{j}^{2}-b_{j}\right| \\
& \leq m^{-1} \sum_{j=1}^{s} A_{j}^{4} N_{j}^{-2-6 \delta}(m / M) N_{j}^{2+4 \delta} A_{j}^{-4}<M^{-1},
\end{aligned}
$$

contradicting our initial hypothesis. This proves the lemma.

Proof of Theorem 1 . We proceed by induction on $s$. Clearly Heilbronn's theorem is equivalent to Theorem 1 when $s=1$. Now suppose that $s>1$ and the result has been proved for forms in $s-1$ variables. It is easily verified that, since $1 / 2 \leq \beta(s) \leq 1 / 2+1 /(2 s-4)$, we have

$$
\sigma(s) \leq 2 \quad \text { and } \quad \frac{s}{s-1} \sigma(s-1) \geq \sigma(s) .
$$

If $N_{j}>N^{\varepsilon / 4}(j=1, \ldots, s)$, then the induction step follows from Lemma 3. Thus we may suppose $N_{j} \leq N^{\varepsilon / 4}$ for some index $j$, let us say $j=s$. Consequently,

$$
N_{1} \ldots N_{s-1} \geq N^{s-\varepsilon / 4} \geq\left(N^{s /(s-1)-\varepsilon / 4}\right)^{s-1} .
$$

By the induction hypothesis there are integers $n_{1}, \ldots, n_{s-1}$, not all zero, satisfying

$$
\begin{gathered}
0 \leq n_{i} \leq N_{i} \quad(i=1, \ldots, s-1), \\
\left\|\alpha_{1} n_{1}^{2}+\ldots+\alpha_{s-1} n_{s-1}^{2}\right\|<N^{-(s /(s-1)-\varepsilon / 4)(\sigma(s-1)-\varepsilon / 4)} \leq N^{-\sigma(s)+\varepsilon} .
\end{gathered}
$$

The last inequality follows from (2.20). This completes the induction step and proves Theorem 1.

3. The lattice method. We write $\boldsymbol{a} \boldsymbol{b}$ for inner product in $\mathbb{R}^{2}$ and $|\boldsymbol{a}|=(\boldsymbol{a} \boldsymbol{a})^{1 / 2}$. The area of the parallelogram spanned by $\boldsymbol{a}$ and $\boldsymbol{b}$ is denoted by $\operatorname{det}(\boldsymbol{a}, \boldsymbol{b})$. Let

$$
K_{0}=\left\{\boldsymbol{x} \in \mathbb{R}^{2}:|\boldsymbol{x}|<1\right\} .
$$

If $S, T \subset \mathbb{R}^{2}$ and $c \in \mathbb{R}$ then $c S$ denotes the set $\{c s: s \in S\}$, and $S+T=$ $\{\boldsymbol{s}+\boldsymbol{t}: \boldsymbol{s} \in S, \boldsymbol{t} \in T\}$.

To facilitate comparison with [19] and [2] we prove the following result in place of Theorem 2 .

Proposition. Let $\varepsilon>0, s \geq 2, N>C_{5}(s, \varepsilon)$ and

$$
\lambda(s)= \begin{cases}1 / 2+2 / s & (s=2,3,4), \\ 4 / s+(1-4 / s) / \sigma(s) & (s=5,6,7), \\ 1 / 2+1 /(2 \sigma(s)) & (s \geq 8) .\end{cases}
$$


Let $\Delta$ be a positive number satisfying

$$
1<\Delta^{\lambda(s)+\varepsilon} \leq N
$$

and let $\Lambda=\Delta^{1 / 2} \mathbb{Z}^{2}$. Then for any $\boldsymbol{\alpha}_{1}, \ldots, \boldsymbol{\alpha}_{s} \in \mathbb{R}^{2}$ there are integers $n_{1}, \ldots, n_{s}$ satisfying (1.2) and

$$
n_{1}^{2} \boldsymbol{\alpha}_{1}+\ldots+n_{s}^{2} \boldsymbol{\alpha}_{s} \in \Lambda+K_{0} .
$$

To deduce Theorem 2, we first note that

$$
\lambda(s)=1 /(2 \tau(s)),
$$

as the reader may easily verify. Let $\Delta=N^{2(\tau(s)-\varepsilon)}$ so that (3.1) holds. Let $\boldsymbol{\alpha}_{j}=N^{\tau(s)-\varepsilon}\left(\alpha_{j}, \beta_{j}\right)$. Then (3.2) implies

$$
\left|N^{\tau(s)-\varepsilon}\left(n_{1}^{2} \alpha_{1}+\ldots+n_{s}^{2} \alpha_{s}\right)-N^{\tau(s)-\varepsilon} m\right|<1
$$

for some integer $m$, and similarly for the $\beta_{j}$. Now Theorem 2 follows at once.

In the same vein we have the following corollary of Theorem 1.

Corollary. Let $s \geq 1, \delta>0$ and $N>C_{4}(s, \delta)$. Suppose that $N_{1}, \ldots$ $\ldots, N_{s}$ satisfy (1.4). Let $S$ be a one-dimensional subspace of $\mathbb{R}^{2}$ and $\Lambda_{1}$ a lattice in $S$ generated by a point $\boldsymbol{z}$ satisfying

$$
|\boldsymbol{z}|<N^{\sigma(s)-\delta} .
$$

Then for any $\boldsymbol{\alpha}_{1}, \ldots, \boldsymbol{\alpha}_{s}$ in $S$ there are non-negative integers $n_{1}, \ldots, n_{s}$, not all zero, satisfying $n_{i} \leq N_{i}$ and

$$
n_{1}^{2} \boldsymbol{\alpha}_{1}+\ldots+n_{s}^{2} \boldsymbol{\alpha}_{s} \in \Lambda_{1}+K_{0} .
$$

In the remainder of the paper, $\Delta, \Lambda$ are as in the Proposition. Let $\Pi$ be the polar lattice of $\Lambda, \Pi=\Delta^{-1 / 2} \mathbb{Z}^{2}$. Let $\Pi^{*}$ be the set of primitive points of $\Pi$. Evidently

$$
|\boldsymbol{p}| \geq \Delta^{-1 / 2} \quad\left(\boldsymbol{p} \in \Pi^{*}\right) .
$$

(Usually the lattice method is applied to general lattices in $\mathbb{R}^{h}$. The righthand side of (3.5) would then be, essentially, $\Delta^{-1}$. The stronger bound (3.5) is crucial to our proof.)

Let $\boldsymbol{p} \in \Pi^{*}$ and let $\boldsymbol{p}^{\perp}=\left\{\boldsymbol{x} \in \mathbb{R}^{2}: \boldsymbol{x p}=0\right\}$. Clearly $2 \Lambda \cap \boldsymbol{p}^{\perp}$ is a lattice in $\boldsymbol{p}^{\perp}$ generated by a point $\boldsymbol{z}$ having

$$
|\boldsymbol{z}|=2 \Delta|\boldsymbol{p}| \text {. }
$$

In our application of the Corollary, we shall have $S=\boldsymbol{p}^{\perp}, \Lambda_{1}=2 \Lambda \cap \boldsymbol{p}^{\perp}$.

Lemma 4. (i) Let $\boldsymbol{p} \in \Pi^{*}$. Any point $\boldsymbol{a} \in \mathbb{R}^{2}$ may be written in the form

$$
a=l+s+b
$$


where $\boldsymbol{l} \in \Lambda, \boldsymbol{s} \in \boldsymbol{p}^{\perp}$ and

$$
|\boldsymbol{b}| \ll|p|^{-1}\|\boldsymbol{p a}\| .
$$

(ii) Let $\boldsymbol{p}_{1}, \boldsymbol{p}_{2}$ be linearly independent points of $\Pi^{*}$. There is a positive integer $c$,

$$
c \ll \operatorname{det}\left(\boldsymbol{p}_{1}, \boldsymbol{p}_{2}\right) \Delta,
$$

such that any $\boldsymbol{a} \in \mathbb{R}^{2}$ may be written in the form

$$
\boldsymbol{a}=c^{-1}(\boldsymbol{k}+\boldsymbol{d})
$$

where $\boldsymbol{k} \in \Lambda$ and

$$
|\boldsymbol{d}| \ll \Delta \max \left(\left|\boldsymbol{p}_{1}\right|,\left|\boldsymbol{p}_{2}\right|\right) \max \left(\left\|\boldsymbol{p}_{1} \boldsymbol{a}\right\|,\left\|\boldsymbol{p}_{2} \boldsymbol{a}\right\|\right) .
$$

Pro of. These are two special cases of Lemma 7.9 of [2].

Lemma 5. Let $\varepsilon>0, N>C_{8}(\varepsilon)$. Let $\mathcal{A}$ be a subset of $\Pi^{*}$ with $|\boldsymbol{p}| \leq N$ for all $\boldsymbol{p}$ in $\mathcal{A}$. Suppose that any two vectors in $\mathcal{A}$ have determinant $\leq Z$. Let $\boldsymbol{e} \in \mathbb{R}^{2}$. Let $U, V$ be positive numbers such that for each $\boldsymbol{p}$ in $\mathcal{A}$ there are coprime integers $q(\boldsymbol{p}), w(\boldsymbol{p})$ having

$$
1 \leq q(\boldsymbol{p}) \leq U \leq N, \quad|q(\boldsymbol{p}) \boldsymbol{e} \boldsymbol{p}-w(\boldsymbol{p})| \leq V .
$$

Suppose further that $\Delta \leq N^{2}$,

$$
Z U^{2} V \Delta N^{\delta} \leq 1 .
$$

Then there is an integer $q$ and a subset $\mathcal{C}$ of $\mathcal{A}$ such that

$$
|\mathcal{C}| \geq|\mathcal{A}| N^{-\delta}, \quad q(\boldsymbol{p})=q \quad \text { for all } \boldsymbol{p} \in \mathcal{C} .
$$

Proof. See [2], Lemma 7.6.

The starting point for the proof of the Proposition is the following variant of Lemma 1.

Lemma 6. Let $\delta>0, N>C_{9}(\delta)$. Let $\boldsymbol{x}_{1}, \ldots, \boldsymbol{x}_{N}$ be a sequence in $\mathbb{R}^{2}$ with

$$
\boldsymbol{x}_{n} \notin \Lambda+K_{0} \quad(n=1, \ldots, N) .
$$

Then

$$
\sum_{\boldsymbol{p} \in \Pi, 0<|\boldsymbol{p}|<N^{\delta}}\left|\sum_{n=1}^{N} e\left(\boldsymbol{p} \boldsymbol{x}_{n}\right)\right| \gg N .
$$

Proof. See [2], Lemma 7.4.

Proof of the Proposition. For brevity, write $\sigma=\sigma(s), \lambda=\lambda(s)$. We may suppose that $0<\varepsilon<1 / 2$. Let $\delta=\varepsilon / 40$, so that

$$
\Delta^{\lambda} \leq N^{1-20 \delta} \text {. }
$$


Suppose that no integers $n_{1}, \ldots, n_{s}$ satisfy (1.2) and (3.2). By Lemma 6 , we have

$$
\sum_{0<|\boldsymbol{p}|<N^{\delta}, \boldsymbol{p} \in \Pi} T_{1}(\boldsymbol{p}) \ldots T_{s}(\boldsymbol{p}) \gg N^{s}
$$

where

$$
T_{i}(\boldsymbol{p})=\left|\sum_{n=1}^{N} e\left(n^{2} \boldsymbol{p} \boldsymbol{\alpha}_{i}\right)\right| .
$$

Since every $\boldsymbol{p} \in \Pi$ is an integer multiple of a primitive point, it follows that

$$
\sum_{|\boldsymbol{p}|<N^{\delta}, \boldsymbol{p} \in \Pi^{*}} S(\boldsymbol{p}) \gg N^{s}
$$

where

$$
S(\boldsymbol{p})=\sum_{t=1}^{\left[N^{\delta} /|\boldsymbol{p}|\right]} T_{1}(t \boldsymbol{p}) \ldots T_{s}(t \boldsymbol{p}) .
$$

We cover the interval $\left[\Delta^{-1 / 2}, N^{\delta}\right)$ with $O(\log N)$ intervals $[a, 2 a)$. In view of (3.5), there is an $a$ satisfying

$$
\begin{gathered}
\Delta^{-1 / 2} \leq a<N^{\delta}, \\
\sum_{\boldsymbol{p} \in \Pi^{*}, a \leq|\boldsymbol{p}|<2 a} S(\boldsymbol{p}) \gg N^{s} / \log N .
\end{gathered}
$$

There are $\ll \Delta a^{2}$ summands here, so that the contribution from $\boldsymbol{p}$ with

$$
S(\boldsymbol{p})<N^{s}(\log N)^{-2} \Delta^{-1} a^{-2}
$$

is negligible. Covering $\left[N^{s}(\log N)^{-2} \Delta^{-1} a^{-2}, a^{-1} N^{s+\delta}\right]$ with $O(\log N)$ intervals $[B, 2 B)$, we see that there is a $B$ with

$$
N^{s}(\log N)^{-2} \Delta^{-1} a^{-2} \leq B<a^{-1} N^{s+\delta}
$$

and a subset $\mathcal{B}$ of $\Pi^{*}$ with

$$
\begin{gathered}
a \leq|\boldsymbol{p}|<2 a, \quad B \leq S(\boldsymbol{p})<2 B \quad \text { for } \boldsymbol{p} \in \mathcal{B}, \\
\sum_{\boldsymbol{p} \in \mathcal{B}} S(\boldsymbol{p}) \gg N^{s}(\log N)^{-2} .
\end{gathered}
$$

It is convenient to write $X=N^{s} B^{-1}$, so that

$$
\begin{gathered}
X \leq \Delta a^{2} N^{\delta}, \\
S(\boldsymbol{p}) \geq N^{s} X^{-1} \quad(\boldsymbol{p} \in \mathcal{B})
\end{gathered}
$$

and clearly

$$
|\mathcal{B}| \gg X N^{-\delta}
$$


Consider the following conditions:

(A) $\quad X \geq N^{5 \delta}$ and $\Delta a X^{2 / s-1 / 2} \leq N^{1-10 \delta}$;

(B) $\quad \Delta^{1 / \sigma} a^{-1+1 / \sigma} X^{2 / s} \leq N^{1-10 \delta} \quad$ if $s \geq 3 ; \quad X<N^{5 \delta} \quad$ if $s=2$.

We will first derive a contradiction provided that (A) or (B) holds. In conclusion we show that one of (A), (B) must be satisfied.

Suppose first that $(\mathrm{A})$ is satisfied. Using $T_{1} \ldots T_{s} \leq T_{1}^{s}+\ldots+T_{s}^{s},(3.17)$ gives

$$
\sum_{t \leq N^{\delta} a^{-1}} T_{i}(t \boldsymbol{p})^{s} \gg N^{s} X^{-1}
$$

for some index $i$ depending on $\boldsymbol{p}$. There are only $s$ possible $i$, and we may assume without loss of generality that (3.19) holds for $i=1$.

We use the inequality

$$
\left(b_{1}^{l}+\ldots+b_{m}^{l}\right)^{1 / l} \leq\left(b_{1}^{r}+\ldots+b_{m}^{r}\right)^{1 / r}, \quad 0<r \leq l,
$$

for positive numbers $b_{j}$ ([12], p. 28). Thus

$$
\sum_{t \leq N^{\delta} a^{-1}} T_{1}(t \boldsymbol{p})^{2} \gg N^{2} X^{-2 / s} \quad(\boldsymbol{p} \in \mathcal{B}) .
$$

We may apply Lemma 2 to the sum in (3.20). To see this,

$$
a^{-1} X^{2 / s} \leq \Delta^{2 / s} a^{2 / s-1} N^{2 \delta} \leq \Delta^{1 / s+1 / 2} N^{2 \delta} \leq \Delta^{\lambda} N^{2 \delta} \leq N^{1-3 \delta}
$$

from (3.16), (3.15), (3.13). Hence

$$
N^{2} X^{-2 / s} \geq N^{1+\delta}\left[a^{-1} N^{\delta}\right] .
$$

The lemma yields natural numbers $q(\boldsymbol{p})$ for each $\boldsymbol{p}$ in $\mathcal{B}$ satisfying

$$
\begin{gathered}
q(\boldsymbol{p}) \ll a^{-1} X^{2 / s} N^{2 \delta}, \\
\left\|q(\boldsymbol{p}) \boldsymbol{p} \boldsymbol{\alpha}_{1}\right\| \ll X^{2 / s} N^{-2+2 \delta} .
\end{gathered}
$$

The next step is to apply Lemma 5 to a suitable subset of $\mathcal{B}$. The $(x, y)$ plane may be covered by $\ll|\mathcal{B}| N^{-2 \delta}$ angular sections centred at $\mathbf{0}$, of angle $|\mathcal{B}|^{-1} N^{2 \delta} \leq X^{-1} N^{4 \delta}$. Here we have used (3.18) and the hypothesis $X \geq$ $N^{5 \delta}$. One of these sections must contain $\gg N^{2 \delta}$ points of $\mathcal{B}$. Let $\mathcal{A}$ be the set of points of $\mathcal{B}$ lying in this section. In the notation of Lemma 5 , we may take

$$
\boldsymbol{e}=\boldsymbol{\alpha}_{1}, \quad Z=a^{2} X^{-1} N^{4 \delta}, \quad U \ll a^{-1} X^{2 / s} N^{2 \delta}, \quad V \ll X^{2 / s} N^{-2+2 \delta}
$$

in view of the definition of $\mathcal{A},(3.21)$ and (3.22). Moreover, by (3.16), (3.15) and condition $(\mathrm{A})$,

$$
\begin{aligned}
Z U^{2} V \Delta N^{2 \delta} & \ll a^{2} X^{-1} N^{4 \delta}\left(a^{-1} X^{2 / s} N^{2 \delta}\right)^{2}\left(N^{-2+2 \delta} X^{2 / s}\right) \Delta N^{2 \delta} \\
& \ll X^{6 / s-1} \Delta N^{-2+12 \delta} \ll \begin{cases}\Delta^{6 / s} N^{-2+30 \delta} & (s \leq 6) \\
\Delta N^{-2+12 \delta} & (s>6)\end{cases} \\
& \ll \Delta^{2 \lambda} N^{-2+30 \delta} \ll 1 .
\end{aligned}
$$


We deduce from Lemma 5 that there is a subset $\mathcal{C}$ of $\mathcal{A}$ of cardinality $\gg N^{\delta}$, and a natural number $q$ such that $q(\boldsymbol{p})=q$ for every $\boldsymbol{p}$ in $\mathcal{C}$.

Let $\boldsymbol{p}_{1}, \boldsymbol{p}_{2}$ be two linearly independent points in $\mathcal{C}$. We apply Lemma 4(ii) with $\boldsymbol{a}$ replaced by $q \boldsymbol{\alpha}_{1}$. There is a natural number $c$ such that

$$
\begin{gathered}
c \ll \operatorname{det}\left(\boldsymbol{p}_{1}, \boldsymbol{p}_{2}\right) \Delta \leq a^{2} X^{-1} N^{4 \delta} \Delta, \\
c q \boldsymbol{\alpha}_{1}=\boldsymbol{k}+\boldsymbol{d}, \quad \boldsymbol{k} \in \Lambda, \\
|\boldsymbol{d}| \ll \Delta a X^{2 / s} N^{-2+2 \delta} .
\end{gathered}
$$

Here we use once again the definition of $\mathcal{A}$ and (3.22).

Now let $n=c q$. By (3.23), (3.21) and condition (A),

$$
1 \leq n \ll a^{2} X^{-1} N^{4 \delta} \Delta a^{-1} X^{2 / s} N^{2 \delta}=a X^{2 / s-1} \Delta N^{6 \delta} \ll N^{1-\delta} .
$$

Moreover,

$$
\begin{gathered}
n^{2} \boldsymbol{\alpha}_{1}=c q \boldsymbol{k}+c q \boldsymbol{d}, \\
|c q \boldsymbol{d}|=n|\boldsymbol{d}| \ll a X^{2 / s-1} \Delta N^{6 \delta} \Delta a X^{2 / s} N^{-2+2 \delta} \\
\ll a^{2} X^{4 / s-1} \Delta^{2} N^{-2+8 \delta} \ll N^{-\delta}
\end{gathered}
$$

by (3.24) and condition (A). Thus $1 \leq n \leq N, n^{2} \boldsymbol{\alpha}_{1} \in \Lambda+K_{0}$. We have reached a contradiction when (A) holds.

Now suppose that condition (B) holds. We deal with the case $s \geq 3$ first. Fix any $\boldsymbol{p}$ in $\mathcal{B}$. From (3.17),

$$
\sum_{t \leq a^{-1} N^{\delta}} T_{1}(t \boldsymbol{p}) \ldots T_{s}(t \boldsymbol{p}) \geq N^{s} X^{-1} .
$$

As in the proof of $(2.8)$, there must be $A_{1}, \ldots, A_{s}$ in $\left[N^{-2}, N\right]$ and a set $\mathcal{A}$ of $t$ having

$$
\begin{gathered}
1 \leq t \leq a^{-1} N^{\delta} \quad(t \in \mathcal{A}), \\
A_{i} \leq T_{i}(t \boldsymbol{p})<2 A_{i} \quad(i=1, \ldots, s ; t \in \mathcal{A})
\end{gathered}
$$

and

$$
|\mathcal{A}|^{2} A_{1}^{2} \ldots A_{s}^{2} \gg N^{2 s-\delta} X^{-2}
$$

We may assume that

$$
A_{1} \geq \ldots \geq A_{s} .
$$

The next step, in which we deduce a good rational approximation to $\boldsymbol{\alpha}_{i} \boldsymbol{p}$, is different according as $i \leq 2$ or $i>2$. By (3.27) and (3.28),

$$
\begin{aligned}
|\mathcal{A}|^{2} A_{2}^{2(s-1)} & \geq N^{2(s-1)-2 \delta} X^{-2}, \\
|\mathcal{A}|^{2 /(s-1)} A_{2}^{2} & \geq N^{2-2 \delta} X^{-2 /(s-1)} .
\end{aligned}
$$


Since $s \geq 3$, this implies for $i=1,2$ that

$$
\sum_{t \leq a^{-1} N^{\delta}} T_{i}(t \boldsymbol{p})^{2} \geq|\mathcal{A}| A_{i}^{2} \geq N^{2-2 \delta} X^{-2 /(s-1)} .
$$

By (3.16), (3.15) and (3.13),

$$
\begin{aligned}
a^{-1} X^{2 /(s-1)} N^{4 \delta} & \leq \Delta^{2 /(s-1)} a^{2 /(s-1)-1} N^{6 \delta} \leq \Delta^{1 / 2+1 /(s-1)} N^{6 \delta} \\
& \leq \Delta^{\lambda} N^{6 \delta} \leq N .
\end{aligned}
$$

Consequently,

$$
N^{1+\delta}\left[a^{-1} N^{\delta}\right] \leq N^{2-2 \delta} X^{-2 /(s-1)} .
$$

In view of (3.30), we may apply Lemma 2 to (3.29), obtaining natural numbers $q_{1}, q_{2}$ having

$$
\begin{gathered}
q_{i} \leq a^{-1} N^{2+2 \delta} A_{i}^{-2}|\mathcal{A}|^{-1} \\
\left\|q_{i}^{2} \boldsymbol{\alpha}_{i} \boldsymbol{p}\right\| \leq q_{i}\left\|q_{i} \boldsymbol{\alpha}_{i} \boldsymbol{p}\right\| \leq a^{-1} N^{2+3 \delta} A_{i}^{-4}|\mathcal{A}|^{-2} .
\end{gathered}
$$

Now pick any $t \in \mathcal{A}$. If $A_{i} \geq N^{1 / 2+\delta}$ for an index $i \geq 3$, we may apply the case $L=1$ of Lemma 2 to $T_{i}(t \boldsymbol{p})$. This gives a natural number $r_{i}$ with

$$
r_{i} \leq N^{2+\delta} A_{i}^{-2}, \quad\left\|r_{i} t \boldsymbol{\alpha}_{i} \boldsymbol{p}\right\| \leq A_{i}^{-2} N^{\delta} .
$$

Writing $q_{i}=r_{i} t$, we then have, from (3.25),

$$
\begin{gathered}
q_{i} \leq a^{-1} N^{2+2 \delta} A_{i}^{-2}, \\
\left\|q_{i}^{2} \boldsymbol{\alpha}_{i} \boldsymbol{p}\right\| \leq a^{-1} N^{2+5 \delta} A_{i}^{-4} .
\end{gathered}
$$

Just as in (2.16), we can in fact find $q_{i}$ satisfying (3.33), (3.34) for every $i=1, \ldots, s$.

Combining (3.31)-(3.34), we have

$$
\begin{gathered}
q_{i} \leq a^{-1} N^{2+2 \delta} A_{i}^{-2} C(i)^{-1}, \\
\left\|q_{i}^{2} \boldsymbol{\alpha}_{i} \boldsymbol{p}\right\| \leq a^{-1} N^{2+5 \delta} A_{i}^{-4} C(i)^{-2},
\end{gathered}
$$

where $C(i)=|\mathcal{A}|$ for $i \leq 2, C(i)=1$ for $i>2$. Applying Lemma 4(i) and (3.36), we obtain

$$
q_{i}^{2} \boldsymbol{\alpha}_{i}=\boldsymbol{l}_{i}+\boldsymbol{t}_{i}+\boldsymbol{b}_{i},
$$

where $\boldsymbol{l}_{i} \in \Lambda, \boldsymbol{t}_{i} \in \boldsymbol{p}^{\perp}$ and

$$
\left|\boldsymbol{b}_{i}\right| \ll a^{-1} a^{-1} N^{2+5 \delta} A_{i}^{-4} C(i)^{-2} \ll a^{-2} N^{2+5 \delta} A_{i}^{-4} C(i)^{-2} .
$$

Recalling (3.6), we apply the Corollary, taking $S=\boldsymbol{p}^{\perp}, \Lambda_{1}=2 \Lambda \cap \boldsymbol{p}^{\perp}$, and replacing $\boldsymbol{\alpha}_{j}$ by $2 \boldsymbol{t}_{j}$ and $N$ by $N^{*}=(a \Delta)^{1 / \sigma} N^{2 \delta}$. We replace $N_{i}$ by

$$
N_{i}^{*}=(a \Delta)^{1 / \sigma} A_{i}^{2} N^{-2+3 \delta} X^{2 / s} C(i) .
$$

We must verify (1.4). From (3.27),

$$
N_{1}^{*} \ldots N_{s}^{*}=(a \Delta)^{s / \sigma}|\mathcal{A}|^{2} A_{1}^{2} \ldots A_{s}^{2} N^{-2 s+3 s \delta} X^{2} \geq\left(N^{*}\right)^{s} .
$$


Thus there are non-negative integers $m_{1}, \ldots, m_{s}$, not all zero, satisfying

$$
\begin{gathered}
2 m_{1}^{2} \boldsymbol{t}_{1}+\ldots+2 m_{s}^{2} \boldsymbol{t}_{s} \in 2 \Lambda+K_{0}, \\
m_{i} \leq N_{i}^{*} \quad(i=1, \ldots, s) .
\end{gathered}
$$

Now let $n_{i}=q_{i} m_{i}$. Not all $n_{i}$ are zero. Moreover, by (3.35), (3.40) and condition (B),

$$
\begin{aligned}
n_{i} & \leq a^{-1} N^{2+2 \delta} A_{i}^{-2} C(i)^{-1}(a \Delta)^{1 / \sigma} A_{i}^{2} N^{-2+3 \delta} X^{2 / s} C(i) \\
& =\Delta^{1 / \sigma} a^{1 / \sigma-1} X^{2 / s} N^{5 \delta} \leq N
\end{aligned}
$$

while

$$
\begin{aligned}
n_{1}^{2} \boldsymbol{\alpha}_{1}+\ldots+n_{s}^{2} \boldsymbol{\alpha}_{s}= & m_{1}^{2} \boldsymbol{l}_{1}+\ldots+m_{s}^{2} \boldsymbol{l}_{s}+m_{1}^{2} \boldsymbol{t}_{1}+\ldots+m_{s}^{2} \boldsymbol{t}_{s} \\
& +m_{1}^{2} \boldsymbol{b}_{1}+\ldots+m_{s}^{2} \boldsymbol{b}_{s} .
\end{aligned}
$$

Here $m_{1}^{2} \boldsymbol{l}_{1}+\ldots+m_{s}^{2} \boldsymbol{t}_{s} \in \Lambda+\frac{1}{2} K_{0}$ by (3.39). By (3.40), (3.38) and condition $(\mathrm{B})$,

$$
\begin{aligned}
\left|m_{i}^{2} \boldsymbol{b}_{i}\right| & \ll(a \Delta)^{2 / \sigma} A_{i}^{4} N^{-4+6 \delta} X^{4 / s} C(i)^{2} a^{-2} N^{2+5 \delta} A_{i}^{-4} C(i)^{-2} \\
& =\Delta^{2 / \sigma} a^{2 / \sigma-2} X^{4 / s} N^{-2+11 \delta} \ll N^{-\delta} .
\end{aligned}
$$

We conclude that

$$
n_{1}^{2} \boldsymbol{\alpha}_{1}+\ldots+n_{s}^{2} \boldsymbol{\alpha}_{s} \in \Lambda+K_{0} .
$$

We have now reached a contradiction when condition (B) holds and $s \geq 3$.

Now let $s=2$. Pick any $\boldsymbol{p} \in \mathcal{B}$. From (3.17) and condition (B) we have

$$
\begin{gathered}
\sum_{t \leq a^{-1} N^{\delta}} T_{1}(t \boldsymbol{p}) T_{2}(t \boldsymbol{p}) \gg N^{2-5 \delta}, \\
\sum_{t \leq a^{-1} N^{\delta}} T_{i}(t \boldsymbol{p}) \gg N^{1-5 \delta} \quad(i=1,2) .
\end{gathered}
$$

From Cauchy's inequality,

$$
\sum_{t \leq a^{-1} N^{\delta}} T_{i}(t \boldsymbol{p})^{2} \gg\left(N^{1-5 \delta}\right)^{2}\left(a^{-1} N^{\delta}\right)^{-1} \gg N^{2-11 \delta} a .
$$

We may apply Lemma 2 , since

$$
N^{2-11 \delta} a\left(N^{\delta} a^{-1}\right)^{-1}=N^{2-12 \delta} a^{2} \gg N^{2-12 \delta} \Delta^{-1} \gg N^{1+\delta}
$$

from (3.15), (3.13). Thus there are natural numbers $q_{1}, q_{2}$ satisfying

$$
\begin{gathered}
q_{i} \ll a^{-2} N^{13 \delta}, \\
\left\|q_{i}\left(\boldsymbol{\alpha}_{i} \boldsymbol{p}\right)\right\| \ll a^{-1} N^{-2+12 \delta} .
\end{gathered}
$$

By Lemma 4(i),

$$
q_{i}^{2} \boldsymbol{\alpha}_{i}=\boldsymbol{l}_{i}+\boldsymbol{s}_{i}+\boldsymbol{b}_{i},
$$


where $\boldsymbol{l}_{i} \in \Lambda, \boldsymbol{s}_{i} \in \boldsymbol{p}^{\perp}$ and

$$
\left|\boldsymbol{b}_{i}\right| \ll a^{-1} q_{i}\left\|q_{i}\left(\boldsymbol{\alpha}_{i} \boldsymbol{p}\right)\right\| \ll a^{-1} a^{-2} N^{13 \delta} a^{-1} N^{-2+12 \delta}=a^{-4} N^{-2+25 \delta} .
$$

Here we used (3.41) and (3.42).

We apply the Corollary as above, this time replacing $N, N_{1}, N_{2}$ by $\Delta a N^{2 \delta}$. There are non-negative integers $m_{1}, m_{2}$, not both zero, with

$$
\begin{gathered}
2 m_{1}^{2} \boldsymbol{s}_{1}+2 m_{2}^{2} \boldsymbol{s}_{2} \in 2 \Lambda+K_{0}, \\
m_{i} \leq \Delta a N^{2 \delta} .
\end{gathered}
$$

Now let $n_{i}=m_{i} q_{i}$. Then

$$
\begin{gathered}
n_{i} \leq \Delta a N^{2 \delta} a^{-2} N^{14 \delta}=a^{-1} \Delta N^{16 \delta} \leq \Delta^{3 / 2} N^{16 \delta} \leq N, \\
\left|m_{i}^{2} \boldsymbol{b}_{i}\right| \ll \Delta^{2} a^{2} N^{4 \delta} a^{-4} N^{-2+25 \delta} \ll a^{-2} \Delta^{2} N^{-2+29 \delta} \ll \Delta^{3} N^{-2+29 \delta} \ll N^{-\delta}
\end{gathered}
$$

from (3.45), (3.43), (3.15) and (3.13). Just as above, we reach a contradiction when $s=2$ and condition (B) holds.

It remains to show that one of $(\mathrm{A}),(\mathrm{B})$ is satisfied. If $X<N^{5 \delta}$ we have, by (3.15) and (3.13),

$$
\Delta^{1 / \sigma} a^{-1+1 / \sigma} X^{2 / s} N^{10 \delta} \leq \Delta^{1 / 2+1 /(2 \sigma)} N^{15 \delta} \leq \Delta^{\lambda} N^{15 \delta} \leq N,
$$

so that (B) holds. If $X \geq N^{5 \delta}$ and $s=2$ then

$$
\Delta a X^{2 / s-1 / 2}=\Delta a X^{1 / 2} \ll \Delta^{3 / 2} a^{2} N^{\delta} \ll \Delta^{3 / 2} N^{3 \delta} \ll N^{1-11 \delta}
$$

from (3.16), (3.15), (3.13), so that (A) holds.

It remains to show that, for $s \geq 3$,

$$
\min \left(\Delta a X^{2 / s-1 / 2} N^{10 \delta}, \Delta^{1 / \sigma} a^{-1+1 / \sigma} X^{2 / s} N^{10 \delta}\right) \leq N
$$

whenever $\Delta^{-1 / 2} \leq a \leq N^{\delta}, N^{5 \delta} \leq X \leq \Delta a^{2} N^{\delta}$.

If $s=3,4$, the left-hand side of (3.46) is

$\leq \Delta a X^{2 / s-1 / 2} N^{10 \delta} \leq \Delta^{1 / 2+2 / s} a^{4 / s} N^{11 \delta} \leq \Delta^{1 / 2+2 / s} N^{13 \delta} \leq \Delta^{\lambda} N^{13 \delta} \leq N$.

If $s=5,6,7$ the left-hand side of (3.46) is

$$
\leq\left(\Delta^{1 / \sigma} a^{-1+1 / \sigma}\right)^{1-4 / s}(\Delta a)^{4 / s} N^{10 \delta} .
$$

The exponent of $a$ here is positive, so we obtain the bound

$$
\leq \Delta^{(1 / \sigma)(1-4 / s)+4 / s} N^{12 \delta}=\Delta^{\lambda} N^{12 \delta} \leq N .
$$

Finally, if $s \geq 8$, the left-hand side of (3.46) is

$$
\begin{aligned}
& \leq\left(\Delta a X^{2 / s-1 / 2}\right)^{1 / 2}\left(\Delta^{1 / \sigma} a^{-1+1 / \sigma} X^{2 / s}\right)^{1 / 2} N^{10 \delta} \\
& \leq \Delta^{1 / 2+1 /(2 \sigma)} a^{1 /(2 \sigma)} X^{2 / s-1 / 4} N^{10 \delta} \\
& \leq \Delta^{1 / 2+1 /(2 \sigma)} N^{11 \delta}=\Delta^{\lambda} N^{11 \delta} \leq N .
\end{aligned}
$$

This completes the proof of the Proposition. 


\section{References}

[1] R. C. Baker, Small solutions of congruences, Mathematika 20 (1983), 164-188.

[2] - Diophantine Inequalities, Oxford University Press, Oxford 1986.

[3] R. C. Baker and J. Brüdern, Pairs of quadratic forms modulo one, Glasgow Math. J., to appear.

[4] R. C. Baker and J. Gajraj, On the fractional parts of certain additive forms, Math. Proc. Cambridge Philos. Soc. 79 (1976), 463-467.

[5] R. C. Baker and G. Harman, Small fractional parts of quadratic and additive forms, ibid. 90 (1981), 5-12.

[6] -, - Small fractional parts of quadratic forms, Proc. Edinburgh Math. Soc. 25 (1982), 269-277.

[7] R. J. Cook, The fractional parts of an additive form, Proc. Cambridge Philos. Soc. 72 (1972), 209-212.

[8] I. Danicic, Contributions to number theory, Ph.D. thesis, London 1957.

[9] —, An extension of a theorem of Heilbronn, Mathematika 5 (1958), 30-37.

[10] - On the fractional parts of $\theta x^{2}$ and $\phi x^{2}$, J. London Math. Soc. 34 (1959), 353357.

[11] - The distribution (mod 1) of pairs of quadratic forms with integer variables, ibid. 42 (1967), 618-623.

[12] G. Hardy, J. E. Littlewood and G. Pólya, Inequalities, Cambridge University Press, Cambridge 1967.

[13] G. Harman, Diophantine approximation and prime numbers, Ph.D. thesis, London 1982.

[14] D. R. Heath-Brown, Small solutions of quadratic congruences II, Mathematika 38 (1991), 264-284.

[15] H. Heilbronn, On the distribution of the sequence $\theta n^{2}(\bmod 1)$, Quart. J. Math. Oxford Ser. (2) 19 (1948), 249-256.

[16] M. C. Liu, On the fractional parts of $\theta n^{k}$ and $\phi n^{k}$, ibid. 21 (1970), 481-486.

[17] —, Simultaneous approximation of two additive forms, Trans. Amer. Math. Soc. 206 (1975), 361-373.

[18] A. Schinzel, H. P. Schlickewei and W. M. Schmidt, Small solutions of quadratic congruences and small fractional parts of quadratic forms, Acta Arith. 37 (1980), 241-248.

[19] W. M. Schmidt, Small fractional parts of polynomials, CBMS Regional Conf. Ser. in Math. 32, Amer. Math. Soc., Providence 1977.

DEPARTMENT OF MATHEMATICS

ROYAL HOLLOWAY AND BEDFORD NEW COLLEGE

EGHAM

SURREY TW20 OEX, U.K.
AM PASTORENHOLZ 8

4972 LÖHNE

GERMANY

Received on 24.6.1991

and in revised form on 26.7.1991 